\title{
Modelling Intergranular Stress Corrosion Cracking in Simulated Three-dimensional Microstructures
}

\author{
A. P. Jivkov ${ }^{1, a}$, N. P. C. Stevens ${ }^{1, b}$ and T. J. Marrow ${ }^{1, c}$ \\ ${ }^{1}$ School of Materials, The University of Manchester, Grosvenor St., Manchester M1 7HS, UK. \\ aandrey.jivkov@manchester.ac.uk, ${ }^{b}$ nicholas.stevens@manchester.ac.uk, \\ james.marrow@manchester.ac.uk
}

Keywords: Stress Corrosion Cracking; Microstructure; Grain boundaries; Crack bridging; Finite element analysis.

\begin{abstract}
Microstructure can have a significant effect on the resistance to intergranular stress corrosion cracking. Certain grain boundaries are susceptible to corrosion while others have high resistance and may form crack bridging ligaments as the crack deviates around them. To investigate the mechanics of crack bridging, 3D computational model has been previously developed. An extension to the model, to include stress corrosion crack growth kinetics is presented in this paper. An analysis of the effects of resistant grain boundary fraction demonstrates that the bridging ligaments can significantly retard short crack propagation rates. Increasing the fraction of resistant boundaries is shown to improve microstructure resistance by reducing the crack propagation rate.
\end{abstract}

\section{Introduction}

Intergranular stress corrosion cracking (IGSCC) is a mode of sub-critical crack propagation in susceptible nickel-based alloys and austenitic stainless steels used in power generation. Experimental results, e.g. [1] for nickel-based alloys and [2] for stainless steels, show that grain boundaries with no special crystallographic relationship between the adjacent grains are more prone to intergranular stress corrosion than certain "special" boundaries, which tend to have high degrees of lattice coincidence. As a simplification, the microstructure can be regarded as containing grain boundaries of two types, which are either resistant or susceptible to corrosion. Based on this binary classification, percolation-like models for assessing failure susceptibility have been proposed (e.g. [3] and [4]). Typically, these use regular microstructure models and the probability of crack advance at triple junctions is based on the grain boundary types and their orientation relative to the applied stress. A drawback is that percolation models do not account for the mechanical crack driving force, which can vary during crack evolution.

Experimental studies have shown that the interaction between a crack and special grain boundaries leads to crack bridging (e.g. [5] and [6]). A crack shielding effect from the bridges is expected to reduce the crack tip strains and thereby retard propagation, particularly for short cracks. Recently, the authors have developed computational finite element based models for studies of intergranular cracking in regular microstructures, using hexagonal unit cell in 2D [7], and truncated octahedron in 3D [8]. These models are capable of simulating bridging ligament formation, crack branching and crack coalescence. The results demonstrated a significant impact of applied mechanical load and crack bridges on crack development, compared to percolation predictions. However, these models did not address the kinetics of intergranular failure, which is essential to quantitatively estimate the microstructure effect on crack growth rates. This paper reports ongoing work to introduce the kinetics of corrosion assisted failure of susceptible boundaries into the 3D model. The model is physically representative of the observed failure mechanisms of grain boundaries in a sensitized material, and the quantitative parameters have yet to be determined experimentally. Selected results for crack growth rates in a study problem are presented and discussed. 


\section{Problem formulation and computational model}

A grain in the model microstructure is represented by a truncated octahedron shown in Fig. 1 (left). This unit cell is convenient as it is topologically close to the real grains and is able to tessellate 3D space compactly. The side of its bounding cube, $D$, is a microstructure length parameter, equivalent to the average grain diameter. The model microstructure is an assembly of unit cells, an example of which is shown in Fig. 1 (centre), with common faces representing the grain boundaries. A sufficiently large assembly is needed to study crack development and the effect of bridges on propagation rate. The assembly used in this work contains 30000 grains with 200000 grain boundaries and occupies the region $\left\{-7.5 D \leq X_{1} \leq 7.5 D,-20 D \leq X_{2} \leq 20 D, 0 \leq X_{3} \leq 25 D\right\}$ with respect to a fixed coordinate system $\left(X_{1}, X_{2}, X_{3}\right)$. A semi-circular pre-crack of radius $0.5 D$, centered at the origin and perpendicular to the $X_{1}$ axis is assumed to initiate crack growth. The model material is a Type 304 austenitic stainless steels, with typical values for Young's modulus $E=193 \mathrm{GPa}$, Poisson's ratio $v=0.29$, proof stress (at $0.2 \%$ plastic strain) $\sigma_{0}=310 \mathrm{MPa}$, and tensile strength (at $60 \%$ elongation) $\sigma_{u}=600 \mathrm{MPa}$. These are taken as the homogeneous mechanical properties of the model, i.e. when the assembly is considered as a continuum. The load is applied via prescribed displacements $u_{1}=-0.00375 D$ at $X_{1}=-7.5 D$ and $u_{1}=0.00375 D$ at $X_{1}=7.5 D$. Further, $u_{3}=0$ at $X_{3}=$ $25 D$, all other boundaries are stress free.
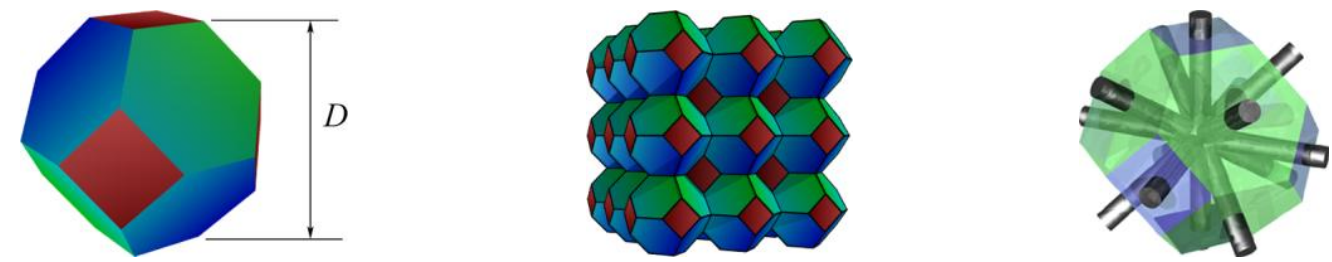

Figure 1. Model microstructure: Solid cell (left); Assembly (centre); Computational cell (right).

The grain boundaries are classified as either resistant (RB) or susceptible (SB) to corrosion. A second microstructure parameter is the RB fraction, $f$, defined as the number of RB divided by the total number of boundaries. Four RB fractions have been considered, $f=0.0,0.20,0.30,0.40$. The microstructure with no $\mathrm{RB}$ is studied for comparative purposes while the others enclose the experimentally determined fractions in microstructures of Type 304 steels [5]. For a given $f$, the boundary types are randomly distributed. The crack is allowed to propagate along grain boundaries only. Simulations are performed with a combination of an in-house code controlling the crack advance and the commercial code Abaqus [9] used as a finite element solver. For the assembly size that is studied, a finite element model with 3D continuum finite elements in the grains is judged as computationally inefficient at present. Instead, a discrete model is used, where nodes are placed in the grains centers and beam elements connect every pair of grains with common boundary. An illustration is given in Fig. 1 (right), and a thorough description is given in reference [8].

Failure of a grain boundary, i.e. a beam element, can occur either via corrosion assisted damage, or via pure mechanical damage. In both cases damage is equivalent to a reduction of the beam element cross section and hence stiffness, and varies from zero (intact boundary) to one (failed boundary). Corrosion assisted damage can occur at SB that are subject to tensile strains and are in contact with the corrosive environment, i.e. the current crack surface. Fig. 2 shows the assumed relationship between the stress corrosion crack growth rate (in $D^{2}$ per unit of time) and the elastic strain energy density. Damage will initiate if the instantaneous strain energy of the boundary is above the threshold and will evolve with the rate described. Fig. 2 is a qualitative description consistent with typical SCC data. The mechanical damage is assumed to initiate at 5\% plastic strain and evolves by linear degradation of the element stiffness towards ultimate failure at $10 \%$ strain. This describes a ductile failure mode, which is valid in principle for all grain boundaries. In the boundary value problem posed above, however, it is expected to become active, if at all, only for RB that develop into crack bridges. This assumption is consistent with experimentally observed grain boundary behaviour (e.g. 
[7] and [8]). The crack growth simulations are performed in a stepwise manner. Each step consists of the solution for the current boundary value problem (Abaqus) and crack advance (in-house code). The crack configuration in each step is described by the crack surface (failed boundaries) and the boundaries in contact with this surface with their current damage. For each SB that is in contact with the crack surface, i.e. liable to corrosion assisted damage, let $A_{i}$ be the total area and $R_{\mathrm{i}}$ the crack growth rate determined using Fig. 2 for the boundary's strain energy, obtained from the mechanical solution. The physical duration of the simulation step is the smallest time interval, $\Delta t=\operatorname{Min}\left\{A_{i} / R_{\mathrm{i}}\right\}$. Each corroding boundary is damaged by reducing its area by the value $\left(R_{\mathrm{i}} \Delta t\right)$. This means that during a simulation step one or more grain boundaries may fail entirely, while the rest are damaged to various degrees. The mechanical damage, which can occur for RB bridges, is time independent.

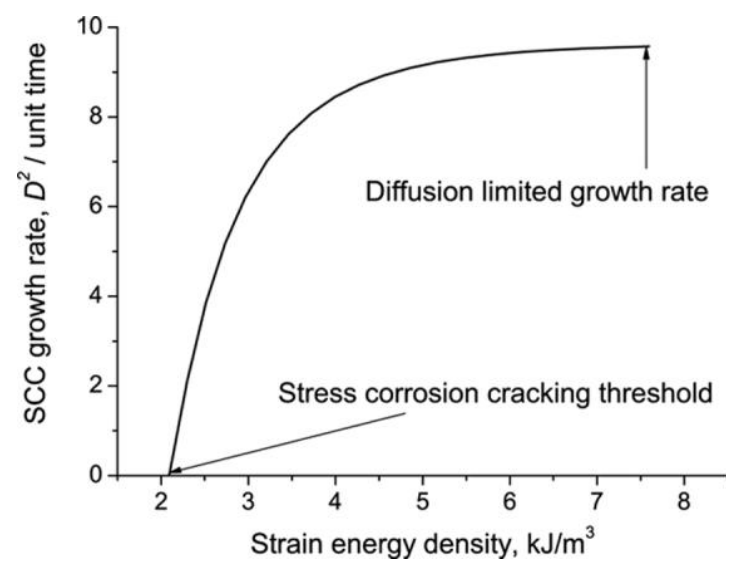

Figure 2. Assumed stress corrosion crack growth rate versus opening mode strain energy density.

\section{Results and discussion}

The morphology of a crack with $f=0.3$ after 50 simulation steps is shown in Fig. 3 . The cracks are approximately semi-circular [8]. The crack surface area, i.e. the total area of the failed and partial areas of the damaged boundaries is approximately $20 D^{2}$. The failed grain boundaries are shown as non-transparent (blue and green in colour). Damaged boundaries have degrees of transparency reciprocal to their damage (red in colour). Holes in the crack surface correspond to bridges.

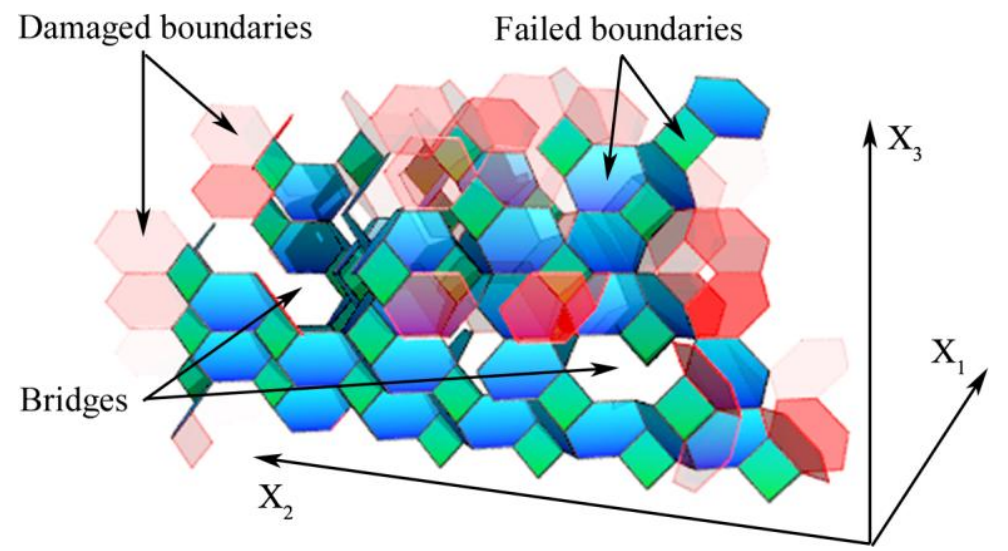

Figure 3. The crack morphology after 50 simulation steps in a microstructure with $f=0.3$.

An example of the calculated crack growth rate at each step, and the resulting average, (in $D^{2}$ per unit of time) is shown in Fig. 4 (left) as a function of crack area (in $D^{2}$ ) after 100 simulation steps in the microstructure with $f=0.3$. The average crack growth rates for the four microstructures are shown in Fig. 4 (right). The calculated crack growth rates are global, i.e. they measure the total increase of crack surface area per time unit. They are not directly equivalent to the local rate described in Fig. 2, as some boundaries may develop damage and fail simultaneously. The time required to propagate a crack to a pre-selected given area of $40 D^{2}$ is also indicated in Fig. 4 . Increasing the fraction of resistant boundaries (RB) decreases the crack growth rate significantly. 

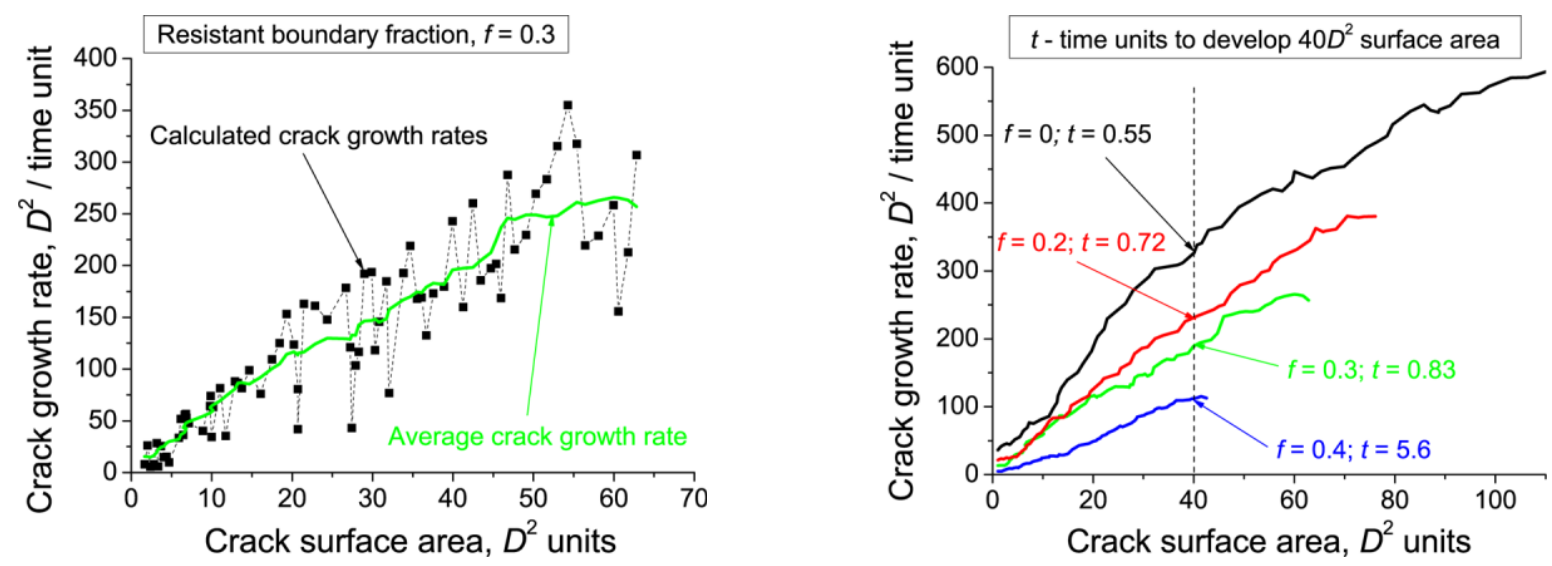

Figure 4. Crack growth rates versus crack area: Rates per step and average for $f=0.3$ (left); Average rates for the four studied microstructures (right).

\section{Conclusions}

A computational model that simulates intergranular crack propagation has been extended to include the failure kinetics of the boundaries that are susceptible to stress corrosion. The results demonstrate that crack tip shielding developed by crack bridging resistant boundaries has a significant effect on the crack propagation rate. Grain boundary engineering to increase the fraction of resistant boundaries is predicted to improve the microstructure resistance to IGSCC by retarding short stress corrosion crack growth rates. Refinements to the model will require suitable data to describe the relationship between the crack growth rate and strain energy (or another equivalent parameter) for susceptible boundaries. This is the subject of ongoing experimental work.

\section{Acknowledgments}

The authors are grateful to Rolls-Royce Plc for the support of APJ, and Nexia Solutions Ltd for the support of NPCS and TJM.

\section{References}

[1] P. Lin, G. Palumbo, U. Erb and K.T. Aust: Scripta Metall. Mater. Vol. 33 (1995), p. 1387.

[2] S.M. Bruemmer and G.S. Was: J. Nuclear Mater. Vol. 216 (1994), p. 348.

[3] G. Palumbo, P.J. King, K.T. Aust, U. Erb and P.C. Lichtenberger: Scripta Metall. Mater. Vol. 25 (1991), p. 1775.

[4] E.M. Lehockey, A.M. Brennenstuhl and I. Thompson: Corros. Sci. Vol. 46 (2004), p. 2383.

[5] T.J. Marrow, L. Babout, A.P. Jivkov, P. Wood, D. Engelberg, N. Stevens, P.J. Withers and R.C. Newman: J. Nuclear Mater. Vol. 352 (2006), p. 62.

[6] L. Babout, T.J. Marrow, D. Engelberg and P.J. Withers: Mater. Sci. Techn. Vol. 22 (2006), p.1068.

[7] A.P. Jivkov, N.P.C. Stevens and T.J. Marrow: Acta Mater. Vol. 54 (2006), p. 3493.

[8] A.P. Jivkov, N.P.C. Stevens and T.J. Marrow: Comput. Mater. Sci. Vol. 38 (2006), p. 442.

[9] ABAQUS User's Manual, Version 6.5 (Abaqus Inc., Providence 2004). 